\title{
Nodular Fasciitis of External Auditory Canal
}

\author{
Jihyun Ahn · Sunyoung Kim \\ Youngsil Park ${ }^{1}$ \\ Departments of Pathology and ${ }^{1}$ Otolaryngology, \\ Dongkang Medical Center, Ulsan, Korea \\ Received: January 28, 2016 \\ Revised: March 8, 2016 \\ Accepted: March 9, 2016 \\ Corresponding Author \\ Jihyun Ahn, MD \\ Department of Pathology, Dongkang Medical \\ Center, 239 Taehwa-ro, Jung-gu, Ulsan 44455, \\ Korea \\ Tel: +82-52-241-1375 \\ Fax: +82-52-241-1366 \\ E-mail: jvcih@hanmail.net
}

Nodular fasciitis is a pseudosarcomatous reactive process composed of fibroblasts and myofibroblasts, and it is most common in the upper extremities. Nodular fasciitis of the external auditory canal is rare. To the best of our knowledge, less than 20 cases have been reported to date. We present a case of nodular fasciitis arising in the cartilaginous part of the external auditory canal. A 19-year-old man complained of an auricular mass with pruritus. Computed tomography showed a $1.7 \mathrm{~cm}$ sized soft tissue mass in the right external auditory canal, and total excision was performed. Histologic examination revealed spindle or stellate cells proliferation in a fascicular and storiform pattern. Lymphoid cells and erythrocytes were intermixed with tumor cells. The stroma was myxoid to hyalinized with a few microcysts. The tumor cells were immunoreactive for smooth muscle actin, but not for desmin, caldesmon, CD34, S-100, anaplastic lymphoma kinase, and cytokeratin. The patient has been doing well during the 1 year follow-up period.

Key Words: Nodular fasciitis; External auditory canal; Ear
Nodular fasciitis is a relatively common benign soft tissue lesion composed of fibroblastic and myofibroblastic proliferation. Since it was first described in 1955 by Konwaler et al., ${ }^{1}$ it remains as the most common benign condition that is confused with sarcoma due to rapid growth, high cellularity, and mitotic activity. ${ }^{2}$ Clinically, nodular fasciitis presents as a rapidly growing mass or nodule in adults between 20 to 40 years of age. It is sometimes accompanied by previous history of trauma. It can cause tenderness, pain, numbness or paresthesia depending on its location. It most frequently occurs in the upper extremities, especially the flexor forearm. Trunk and head and neck are also common sites for nodular fasciitis. ${ }^{2-5}$ However, nodular fasciitis of the auricular region is unusual. ${ }^{3,4}$ Here, we present a rare case of nodular fasciitis of external auditory canal.

\section{CASE REPORT}

A 19-year-old man visited our hospital because of an auricular mass. The mass was present for about 4 weeks and it was accompanied by itching sensation. A previous history of trauma was not noted. A computed tomographic examination showed a $1.7 \mathrm{~cm}$ sized mass on the posterior wall of the cartilaginous portion of the right external auditory canal (Fig. 1). The mass showed soft tissue density and diffuse enhancement on the contrast image. Destruction of the adjacent bone was not identified. Total mass excision was performed. Grossly, the mass was soft, grayish tan, and relatively myxoid. On light microscopy, the mass was relatively well circumscribed without encapsulation and located in the subcutis and dermis. The mass encircled the cartilage but did not invade it. The mass consisted of spindle to stellate cells with minimal nuclear atypia in myxoid to hyalinized stroma. The tumor cells were arranged in a fascicular or storiform pattern and intermixed with scattered lymphoid cells and red blood cells extravasation (Fig. 2A, B). Microcystic changes were noted in more myxoid areas. Mitotic counts were up to 3 per 10 high power fields. Atypical mitosis was not identified. On immunohistochemistry, the tumor cells were positive for smooth muscle actin, but negative for desmin and caldesmon (Fig. 2C). These findings indicated these cells were fibroblasts or myofibroblasts rather than smooth muscle cells. The tumor cells were also negative for S-100, CD34, anaplastic lymphoma kinase (ALK), and cytokeratin. The tumor cells showed cytoplasmic staining for $\beta$-catenin, but nuclear staining was not seen. Based on all these features, we diagnosed this tumor as nodular fasciitis. 


\section{DISCUSSION}

Thompson et al. ${ }^{3}$ reported the largest number of cases of auricular nodular fasciitis. They described 50 cases of auricular nodular fasciitis and its clinicopathologic characteristics. In their 50 cases, 37 cases $(74 \%)$ were located in preauricular and postauricular region. Six cases (12\%) were located in external auditory canal. Auricular nodular fasciitis accounted for $1.5 \%$ of all 3,930 cases of nodular fasciitis in all anatomical sites and for $1.9 \%$ of all 2,930 cases of benign and malignant auricular neoplasms and soft tissue reactive condition identified from 1970 to 1990 at their institution. Auricular nodular fasciitis

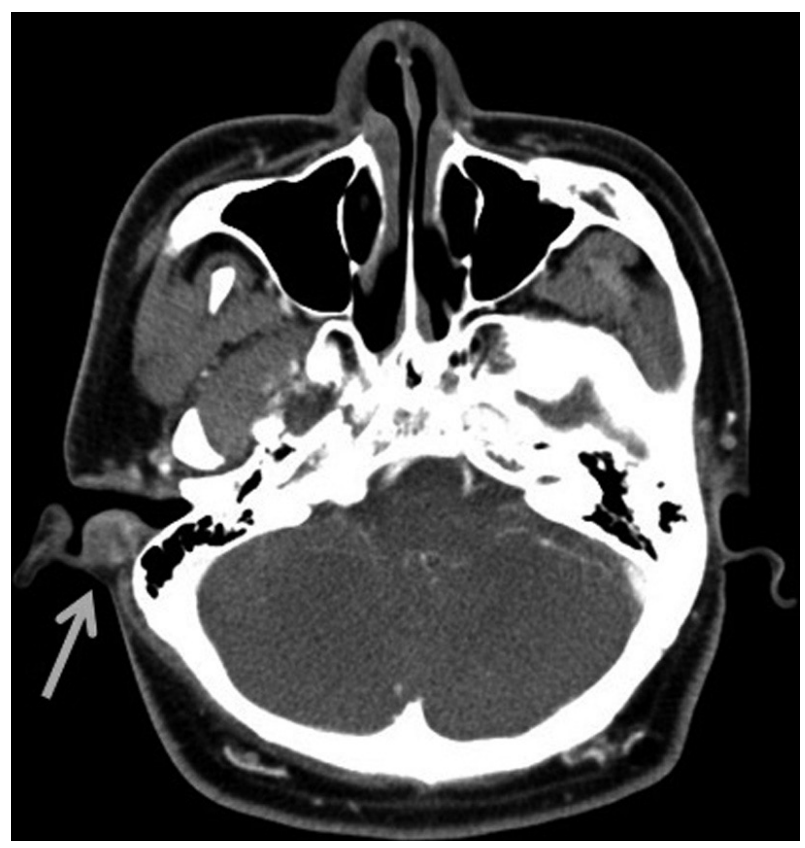

Fig. 1. Radiologic finding. An ovoid mass with soft tissue density (arrow) is noted in the right external auditory canal on computed tomography. was frequently found in young patients, similar to nodular fasciitis elsewhere. Since it was more superficially and dermally located than the one in the extremity, it may present with ulceration and/or bleeding. One of the 50 cases presented with conductive hearing loss. Lesions in the external auricular canal had smaller size (mean, $1.3 \mathrm{~cm}$ ) and a shorter duration of symptoms (mean, 1.6 months) than lesions with a mean size of 1.9 $\mathrm{cm}$ and a mean duration of symptoms of 5.0 months among the total 50 cases. It seems to be due to the narrow space in the external auricular canal. Thompson et al. ${ }^{3}$ found that auricular nodular fasciitis had a higher tendency of local recurrence $(9.3 \%)$ when compared to the recurrence rate of $1 \%-2 \%$ at other sites. ${ }^{2}$ They explained that this resulted from increased auricular trauma and difficulty in complete excision due to the anatomical position.

Auricular nodular fasciitis has similar findings to those of the lesions arising at other common sites. It is composed of fibroblasts or myofibroblasts arranged in a storiform or fascicular pattern with varying cellularity. Most fibroblastic cells are positive for smooth muscle actin, but negative for desmin and caldesmon. Immunostains for S-100, keratin, CD34, ALK, and p53 are also negative. Cytoplasmic staining for $\beta$-catenin can be observed in one-third of the cases. ${ }^{5}$

Nodular fasciitis may be confused with other benign and malignant spindle cell lesions mentioned below.

Inflammatory myofibroblastic tumor shows myofibroblastic proliferation with mixed inflammatory cell infiltrate. Although ALK may be expressed in some cases of inflammatory myofibroblastic tumor, nodular fasciitis does not show ALK expression. ${ }^{6}$

Fibromatosis is usually a large tumor that infiltrates the surrounding soft tissue. Nuclear $\beta$-catenin staining is the characteristic finding of fibromatosis. ${ }^{2,5,7}$
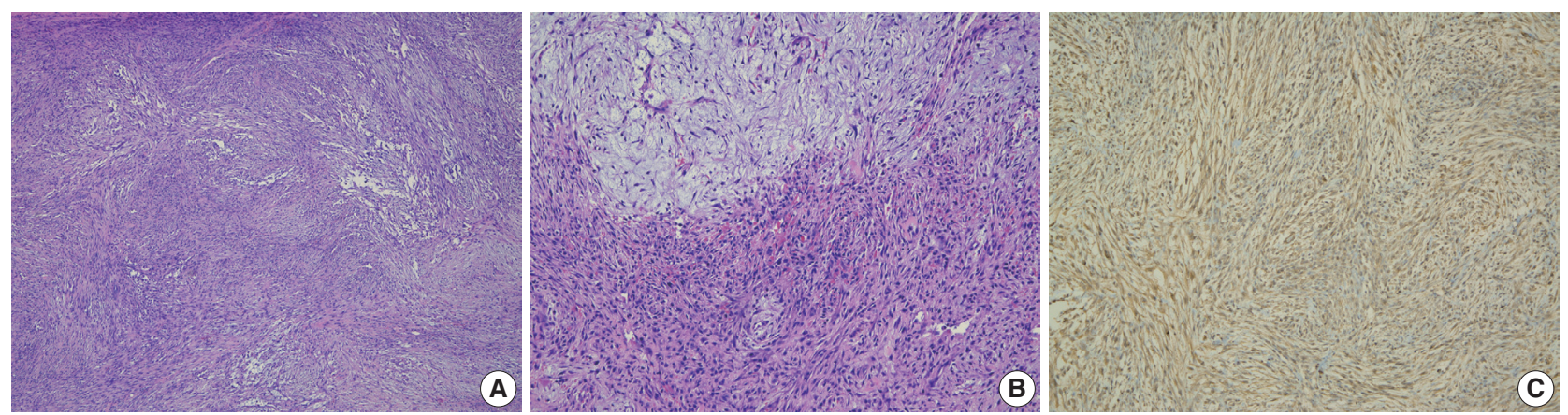

Fig. 2. Pathologic findings. (A) Spindle cell proliferation shows a vaguely storiform to fascicular pattern. (B) The bland spindle and stellate cells are set in loose myxoid (upper portion) to hyalinized matrix (lower portion). Extravasated red blood cells and scattered lymphoid cells are identified. (C) The tumor cells are positive for smooth muscle actin. 
Benign fibrous histiocytoma is typically located in the dermis and has prominent xanthoma cells and occasional Touton-type giant cells. ${ }^{8}$

Solitary fibrous tumor can also occur in the auricular region. Solitary fibrous tumor consists of oval or spindle cells in a fascicular, whorled or haphazard arrangement. Immunohistochemically, the tumor cells are positive for $\mathrm{CD} 34, \mathrm{Bcl}-2$, and CD99. ${ }^{\text {? }}$

Neurogenic tumor shows characteristic wavy, narrow, and elongated cells with S-100 immunoreactivity. Since nodular fasciitis is usually a small sized lesion and does not show prominent nuclear atypia, necrosis, or atypical mitosis, it can be distinguished from malignant lesions.

Nodular fasciitis has been considered as a reactive process and a self-limiting condition, although it has a rare possibility of recurrence. ${ }^{10,11}$ Recently, a few reports reported about the identification of MYH9-USP6 fusion gene in nodular fasciitis. It provided evidence for clonal neoplastic origin of nodular fasciitis and suggested a new model of "transient neoplasia." ${ }^{2-14}$ Also, it showed that the detection of MYH9-USP6 fusion gene can be used as a diagnostic tool for nodular fasciitis. ${ }^{13,14}$ Further investigations related to the molecular analysis of nodular fasciitis are needed.

Here, we presented an unusual case of nodular fasciitis arising in the external auditory canal. Nodular fasciitis must be considered in the differential diagnosis of spindle cell lesions of the external auricular canal. Nodular fasciitis seems to recur more frequently in the auricular region than in the other common sites. Complete excision is likely to be helpful in reducing the recurrence rate.

\section{Conflicts of Interest}

No potential conflict of interest relevant to this article was reported.

\section{REFERENCES}

1. Konwaler BE, Keasbey L, Kaplan L. Subcutaneous pseudosarco- matous fibromatosis (fasciitis). Am J Clin Pathol 1955; 25: 241-52.

2. Weiss SW, Goldblum JR. Enzinger and Weiss's soft tissue tumors. 5th ed. St. Louis: Mosby, 2008.

3. Thompson LD, Fanburg-Smith JC, Wenig BM. Nodular fasciitis of the external ear region: a clinicopathologic study of 50 cases. Ann Diagn Pathol 2001; 5: 191-8.

4. Abdel-Aziz M, Khattab H, El-bosraty H, El-hoshy H, Hesham A, Al-taweel HW. Nodular fasciitis of the external auditory canal in six Egyptian children. Int J Pediatr Otorhinolaryngol 2008; 72: 6436.

5. Peng WX, Kudo M, Yamamoto T, et al. Nodular fasciitis in the parotid gland: a case report and review of the literature. Diagn Cytopathol 2013; 41: 829-33.

6. Jung KH, Kim YW, So YK, Choi SI, Baek MJ. Inflammatory myofibroblastic tumor involving ear lobule. Auris Nasus Larynx 2012; 39: 631-3.

7. Bhattacharya B, Dilworth HP, Iacobuzio-Donahue C, et al. Nuclear beta-catenin expression distinguishes deep fibromatosis from other benign and malignant fibroblastic and myofibroblastic lesions. Am J Surg Pathol 2005; 29: 653-9.

8. Morrissey G, Robinson AC, Stirling R. Cellular benign fibrous histiocytoma of the external auditory meatus. J Laryngol Otol 1996; 110: 98-100.

9. Rezk S, Yousef M, Zamansky M, Khan A. Solitary fibrous tumor of the auditory canal. Arch Pathol Lab Med 2004; 128: e169-71.

10. Kim JR, Chi JG. Nodular fasciitis (13 cases analysis). Korean J Pathol 1998; 22: 190-4.

11. Meng GZ, Zhang HY, Zhang Z, Wei B, Bu H. Myofibroblastic sarcoma vs nodular fasciitis: a comparative study of chromosomal imbalances. Am J Clin Pathol 2009; 131: 701-9.

12. Erickson-Johnson MR, Chou MM, Evers BR, et al. Nodular fasciitis: a novel model of transient neoplasia induced by MYH9-USP6 gene fusion. Lab Invest 2011; 91: 1427-33.

13. Amary MF, Ye H, Berisha F, Tirabosco R, Presneau N, Flanagan AM. Detection of USP6 gene rearrangement in nodular fasciitis: an important diagnostic tool. Virchows Arch 2013; 463: 97-8.

14. Oliveira AM, Chou MM. USP6-induced neoplasms: the biologic spectrum of aneurysmal bone cyst and nodular fasciitis. Hum Pathol 2014; 45: 1-11. 